\title{
A cross-sectional study of skin cancer secondary prevention in rural general practice
}

Scott Kitchener, Janani Pinidiyapathirage, Keegan Hunter

\section{Background and objective \\ As a result of the lack of evidence of reduction in mortality, secondary prevention of melanoma and keratinocyte skin cancers (KSCs) with active screening is not recommended except for individuals at high risk. Limited access to medical professionals for diagnosis and specialised treatment of skin cancers are related to poorer health outcomes for Australians living in rural areas. The aim of this study was to evaluate the secondary prevention of skin cancer in general practice.}

\section{Methods}

A clinical audit of 317 melanomas and $5463 \mathrm{KSCs}$ was conducted, examining the nature of consultation and stage of cancer at diagnosis.

\section{Results}

It was more common for an uncomplicated melanoma $\leq 1 \mathrm{~mm}$ in size to be identified by a general practitioner (GP) during a planned whole-body skin examination than to be the presenting complaint found by the patient $\left(\chi^{2}=4.2, P<0.05\right)$. Half (49\%) of KSCs found were the presenting complaint, with $21 \%$ an incidental diagnosis during another presentation and $30 \%$ found during whole-body skin examinations. Half of all squamous cell carcinomas found were at the intraepidermal stage.

\section{Discussion}

Secondary prevention of skin cancer by GPs is a value proposition for rural populations.
THE CANCER COUNCIL recognises that early diagnosis of skin cancers is related to better outcomes. However, in the absence of sufficient evidence of a significant reduction in mortality, secondary prevention by population-based screening for melanoma has previously been not recommended, with the exception of opportunistic screening by general practitioners (GPs) for patients at high risk. ${ }^{1}$ Evidence shows that whole-body clinical skin examination finds a significant proportion of melanomas and will reduce incidence of thick melanomas at diagnosis..$^{2-4}$ New guidelines for the detection of melanoma and monitoring for individuals at high risk of melanoma have recently been released in Australia, including recommendations for routine surveillance of patients at high risk in addition to training and use of dermoscopy. ${ }^{5}$

The Cancer Council does not recommend routine screening for keratinocyte skin cancers (KSCs) other than for patients who are immunosuppressed or those with a previous KSC, and it is unlikely that secondary prevention with widespread screening will ever be recommended. ${ }^{1}$ For secondary prevention through population-based screening to be recommended, it must reduce mortality. ${ }^{1}$

Mortality from melanoma in Australia was estimated to be 1770 in 2016 , with a further 560 Australians dying of squamous cell carcinoma (SCC) of the skin. ${ }^{6}$ KSCs are the most commonly diagnosed cancers in Australia. With an increasing incidence rate, KSCs contribute significantly to morbidity and mortality, resulting in the highest cancer-related healthcare expenditure in the country. ${ }^{6,7}$ As GPs will be aware, SCCs have an intra-epidermal phase that can be identified early. While basal cell carcinomas do not have an identifiable intra-epidermal phase, delay in diagnosis and management leads to more complicated cancers requiring more complex management beyond general practice.

Early identification of all skin cancers enables improved patient outcomes and results in a wider range of treatment options being available locally in general practice. This is particularly relevant for Australians living in rural areas who would otherwise need to travel for higherlevel treatment required when delayed diagnosis occurs. Limited access to medical professionals for diagnosis and specialised treatment options are contributing factors to the long-standing regional and remote disadvantage for cancer deaths. ${ }^{8}$ Secondary prevention of skin cancers potentially would be a more valuable clinical proposition if cancers were identified in clinically significant earlier stages to enable local treatment options.

The objective of this cross-sectional study was to evaluate secondary prevention of skin cancers as practised in rural general practice. 


\section{Methods}

This study was conducted using a clinical record audit of skin cancer cases identified by billing records in rural general practices in seven rural communities on the Darling Downs over a six-year period (2010-15) for melanoma and three-year period (2013-15) for KSCs. The communities included Clifton, Warwick, Pittsworth, Millmerran, Kingsthorpe, Oakey and Goondiwindi. The demographics of this study population are typical of rural communities in southern Queensland. Agriculture is the largest industry in the region. These sites were chosen as they are small (Australian Statistical Geographical Classification - Remoteness Area [ASGC-RA] Modified Monash Model [MMM] 4-5) centres dependent on healthcare generally delivered by primary care providers with only regional specialist referral access. ${ }^{9}$ The Darling Downs has a greater age standardised incidence rate (ASIR) of melanoma (87.2/100,000 people) than the general Australian population (49.3/100,000) and the Queensland ASIR of melanoma (73.3/100,000)..$^{10,11}$ Demographics of these communities as statistical areas (Statistical Area Level 2) are included in Table 1.
Cases were identified by billing records using specific Medicare Benefits Schedule item numbers for the definitive surgical management of skin cancers and for biopsy of skin lesions at the participating practices. Identified potential cases were included on review of linked electronic clinical records (ECRs); they were confirmed and typed using histology reports returned from specialist pathologists included in the ECR. The manner in which melanomas and KSCs were diagnosed was additionally recorded when available. Individual cancers identified by this methodology more than once were only counted once. Skin cancer type was categorised using terminology employed by reporting histopathologists in addition to histological thickness and invasiveness at diagnosis.

The primary inclusion criterion was histologically confirmed skin cancer identified at the selected participating practices during the respective study periods. Further inclusion criteria for analysis of the circumstances in which diagnosis was made were ECRs detailing the nature of these consultations, and histology specifying depth of skin cancer at diagnosis (for melanoma) and stage of invasion (for SCC). Cases were otherwise excluded for this analysis.
The statistical analyses performed were descriptive and analytic comparisons of the nature of presentation of skin cancers using chi-square tests and odds ratios.

The study was approved by The Royal Australian College of General Practitioners (RACGP) National Research and Evaluation Ethics Committee (NREEC; approval number 16-006) and supported by funding from the Rural Industries Research and Development Corporation and the Skin Cancer College of Australasia.

\section{Results}

\section{Melanoma}

Overall, 317 melanoma cases were identified and confirmed, with the associated clinical circumstances reviewed. Table 2 details 279 melanomas (88\%) reported as uncomplicated and $\leq 1 \mathrm{~mm}$ in thickness, including 140 (44\%) melanomas in situ. Records indicated that 273 cases $(86 \%)$ were managed locally, of whom 27 were patients with melanomas $>1 \mathrm{~mm}$ in thickness at diagnosis. These patients were generally older (mean age 78 years [standard deviation $=12$ years], compared with 65 years [standard deviation $=15$ years] for those with melanomas $\leq 1 \mathrm{~mm}$ in thickness). No

Table 1. Demographic description of the communities studied ${ }^{14}$

\begin{tabular}{|c|c|c|c|c|c|}
\hline Township (SA2) & Population & Female sex & $\mathrm{Age}^{*}$ & Employed (F/T) & Income ${ }^{\dagger}$ \\
\hline Clifton (Clifton-Greenmount) $)^{\ddagger}$ & 4,899 & $49.6 \%$ & 45 & $59.3 \%$ & $\$ 1,141$ \\
\hline Goondiwindi & 6,355 & $50.7 \%$ & 36 & $62.8 \%$ & $\$ 1,391$ \\
\hline Kingsthorpe (Gowrie) & 1,867 & $50.9 \%$ & 37 & $59.8 \%$ & $\$ 1,335$ \\
\hline Pittsworth & 5,722 & $50.6 \%$ & 42 & $62.3 \%$ & $\$ 1,293$ \\
\hline Warwick ${ }^{\ddagger}$ & 15,130 & $51.9 \%$ & 41 & $55.1 \%$ & $\$ 1,007$ \\
\hline Southern Downs East & 4,227 & $49.6 \%$ & 47 & $59.1 \%$ & $\$ 1,027$ \\
\hline
\end{tabular}

*Median age in years

${ }^{\top}$ Median weekly household income

¥The practices at Warwick and Clifton service the areas of Southern Downs West and East.

F/T, full time; SA2, Statistical Area Level 2

These data are reproduced from the Australian Bureau of Statistics (ABS) 2017. 
recurrences from locally managed cases were identified in records at least one year and up to seven years postoperatively.

The reason for the initial consultation could be determined in 282 cases of melanoma (Table 2). Opportunistic skin checks identified 51 (18\%) of melanoma cases. For 115 (41\%) cases, the melanoma was a lesion of concern leading to examinations identified 116 (41\%) of melanomas found. It was more common for an uncomplicated melanoma $\leq 1 \mathrm{~mm}$ in size to be identified by a GP during a planned whole-body skin examination (107/116) than to be the presenting complaint found by the patient $(96 / 115$; $\chi^{2}=4.2, P<0.05$; odds ratio: 2.35 ; risk ratio: 1.11 ).

\section{Keratinocyte skin cancers}

A larger number of KSCs (5463) were confirmed and managed locally by GPs (Table 2). An average of $1.9 \mathrm{KSCs}$ per individual were found, including 2985 SCCs, 2179 basal cell carcinomas and 294 keratoacanthomas. Half (1492) of SCCs were diagnosed and managed at the intra-epidermal stage. In 3504 cases of KSC, the reason for presentation was identifiable from clinical records. The lesions subsequently diagnosed as a KSC were the presenting complaint in half of the cases (1733, 49\%). In 724 (21\%) cases, presentation. Planned whole-body skin

the KSC was an incidental diagnosis during a presentation for an unrelated condition, while 1047 (30\%) of KSCs were found during whole-body skin examinations.

\section{Discussion}

One of the main limitations of this study is the selection of practices on the Darling Downs, which may not be generalisable to rural populations nor models of general practice in other parts of rural Australia. Otherwise, the methods have a number of strengths over previous methods of studying skin cancer including: reasons for consultations were able to be identified in many cases, direct histopathology findings were recorded for all included cases and subsequent management was able to be reviewed. Selection bias was managed by including cases referred for management after identification in the practice. The study focuses on the model of care of general practice; however, some residents of these communities will have travelled to regional centres for identification and management of their skin cancers, so the findings cannot be used to determine the incidence of skin cancer. The sample size has been sufficient to show the benefits of this model of general practice in secondary prevention of skin cancer. Whether the health economic benefit of secondary prevention for skin cancer from

Table 2. Skin cancers identified in general practices of the communities studied

\begin{tabular}{lrr}
\hline & Melanoma & Keratinocyte cancer \\
\hline Total reviewed & 317 & 5,463 \\
\hline Cancers $\leq 1$ mm in thickness & $279(88 \%)$ & $\mathrm{N} / \mathrm{A}$ \\
\hline In situ (melanoma and SCC) & $140(44 \%)$ & $1,492(50 \%)$ \\
\hline SCC & $\mathrm{N} / \mathrm{A}$ & 2,985 \\
\hline Basal cell carcinoma & $\mathrm{N} / \mathrm{A}$ & 2,179 \\
\hline Keratoacanthoma & $\mathrm{N} / \mathrm{A}$ & 294 \\
\hline Reason for presentation detailed in ECR & 282 & 3,504 \\
\hline Lesion = presenting complaint & $115(41 \%)$ & $1,733(49 \%)$ \\
\hline Opportunity/incidental finding & $51(18 \%)$ & $724(21 \%)$ \\
\hline Planned skin check finding & $116(41 \%)$ & $1,047(30 \%)$ \\
\hline
\end{tabular}

ECR, electronic clinical record; N/A, not applicable; SCC, squamous cell carcinoma the general practice model is recognised as significant is for discussion and further investigation.

In these practices, in this model of care, secondary prevention has been effective in identifying a high proportion of skin cancers early ( $88 \%$ of melanomas identified as uncomplicated and $\leq 1 \mathrm{~mm}$ in thickness, and $50 \%$ of SCCs found at the intra-epidermal stage), enabling better patient outcomes and local management to be achieved. These practices are in locations without other specialist services, and the GPs have undertaken further studies in dermoscopy and surgical management of skin cancers to be able to offer such treatment locally.

The nature of presentation of potentially early diagnosable skin cancers is interesting. There are no comparative studies regarding presentations leading to a diagnosis of KSC. In this audit, half of all KSCs were found when patients presented with concerns about skin cancer to be diagnosed by the GP. Another third of cancers were found during planned whole-body skin examinations. Diagnoses by opportunistic screening were only a minority of KSC diagnoses. Half of all SCCs were found prior to invasion, in the intra-epidermal stage. Whole-body skin examinations for screening patients and the accessibility of these practices for patients to present early in the course of KSC development have been effective in moving the stage of SCC found towards earlier diagnosis. Finding intra-epidermal carcinoma has additional clinical significance as it allows GPs to offer topical chemotherapy or photobiological treatment options in the local community. This is a major benefit for rural patients who have less access to other specialist services. It may be beneficial for the RACGP Guidelines for preventive activities in general practice ${ }^{12}$ (Red Book) to recognise this efficacy and these benefits for rural patients when recommending screening for KSCs.

Of significant note for secondary prevention of skin cancer is that planned whole-body skin examinations were associated with identification of melanomas of lesser thickness. Previously, planned whole-body examinations have been found to be associated with 
a $38 \%$ higher chance of identifying a melanoma $<2 \mathrm{~mm}$ in thickness. ${ }^{3}$ With these new findings showing that GPs have an approximately $11 \%$ higher chance of finding thin ( $\leq 1 \mathrm{~mm}$ ) uncomplicated melanomas with planned skin checks, the statistical significance assumes clinical significance. Uncomplicated melanomas of these thicknesses are suitable for local management without the requirement for referral for sentinel node biopsy. ${ }^{13}$ This is of greater real-life significance for people living in rural areas as they may then have access to appropriate surgical care without having to travel to other specialist care. Future editions of the RACGP Red Book ${ }^{12}$ could recognise that this model of general practice, when enacted by GPs with additional training in skin cancer diagnosis and management, is effective at finding melanoma at early stages. This is clinically significant as it enables local management, which is particularly important to those communities without ready access to referred treatment services.

\section{Conclusion}

The overall success of local treatment of skin cancer by rural GPs should be investigated further. However, these are significant findings in terms of patient access to care and overall healthcare costs associated with management of patients locally in primary care as a result of successful secondary prevention.

These findings show that skin cancer identification is an efficient and effective model of care provided in general practice. Additionally, the audit supports implementation of GP-based routine skin examinations for early identification and management of melanoma and SCC both for improved patient outcomes and facilitation of local management. Considering the additional burden of access to care as a factor in rural cancer care disadvantage, there is a stronger case for secondary prevention of skin cancer in rural communities.

Mortality prevented is the conventional method used for determining the worth of secondary prevention programs. When improved patient outcomes from earlier diagnosis and improved access to local treatment options are added to the assessment, the scales may tip in favour of rural general practice, and potentially other general practice settings with less access to specialist care, as worthy of recognition as effective secondary prevention programs.

\section{Authors}

Scott Kitchener MBBS, MD, DrPH, FAFPHM, Professor of Rural Medicine, School of Medicine, Griffith University, Qld. s.kitchener@griffith.edu.au Janani Pinidiyapathirage MBBS, PhD, Research Fellow, Centre for Health, Informatics and Economic Research, University of Southern Queensland, QId Keegan Hunter MD, Junior House Officer, Critical Care Medicine, Gold Coast University Hospital, Qld Competing interests: None.

Funding: SK received a salary through Griffith University. JP received a salary through the University of Southern Queensland. KH received grant funding from the Rural Industries Research and Development Corporation (RIRDC). The Skin Cancer College of Australasia contributed grant funding to support this research.

Provenance and peer review: Not commissioned, externally peer reviewed.

\section{References}

1. Cancer Council Australia's National Skin Cancer Committee. National cancer control policy. Position statement - Early Screening of skin cancer. Sydney, NSW: Cancer Council Australia, 2019. Available at wiki.cancer.org.au/policy/Position_statement_Screening_and_early_detection_of_skin_cancer [Accessed 24 January 2020].

2. Macpherson M, Elwood M, English DR, Baade PD Youl PH, Aitken JF. Presentation and detection of invasive melanoma in a high-risk population. J Am Acad Dermatol 2006;54(5):783-92. doi: 10.1016/j. jaad.2005.08.065.

3. Aitken JF, Elwood M, Baade PD, Youl P, English D. Clinical whole-body skin examination reduces the incidence of thick melanomas. In J Cancer 2010;126(2):450-58. doi: 10.1002/ijc.24747.

4. Youl PH, Baade PD, Parekh S, English D, Elwood M, Aitken JF. Association between melanoma thickness, clinical skin examination and socioeconomic status: Results of a large population-based study. Int J Cancer 2011;128(9):2158-65. doi: 10.1002/ijc.25540.

5. Adler NR, Kelly JW, Guitera P, et al. Methods of melanoma detection and of skin monitoring for individuals at high risk of melanoma: New Australian clinical practice guidelines. Med J Aust 2018;210(1):41-47. doi: 10.5694/mja2.12033.

6. Australian Institute of Health and Welfare. Skin cancer in Australia. Cat. No. CAN 96. Canberra, ACT: AlHW, 2016.

7. Fransen M, Karahalios A, Sharma N, English DR Giles GG, Sinclair RD. Non-melanoma skin cancer in Australia. Med J Aust 2012;197(1):565-68. doi: 10.5694/mja12.10654.

8. Coory MD, Ho T, Jordan SJ. Australia is continuing to make progress against cancer, but the regional and remote disadvantage remains. Med J Aust 2013;199(9):605-80. doi: 10.5694/mja13.10055.

9. Darling Downs Hospital and Health Service. Darling Downs health. Toowoomba City, Qld: Queensland Health, 2019. Available at www.health.qld.gov.au/ darlingdowns/home [Accessed 24 January 2020].
10. Australian Institute of Health and Welfare. Cancer statistics for small geographic areas. Canberra: AlHW, 2019. Available at www.aihw. gov.au/reports/cancer/cancer-statistics-forsmall-geographic-areas/contents/summary [Accessed 24 January 2020].

11. Australian Institute of Health and Welfare. Australian cancer incidence and mortality (ACIM) books: Melanoma of the skin. Canberra, ACT: AlHW, 2017.

12. The Royal Australian College of General Practitioners. Guidelines for preventive activities in general practice. 9th edn, updated. East Melbourne, Vic: RACGP, 2018.

13. Gershenwald JE, Scolyer RA, Hess KR, et al. Melanoma staging: Evidence-based changes in the American Joint Committee on Cancer, 8th edition cancer staging manual. CA Cancer J Clin 2017;67(6):472-92. doi: 10.3322/caac.21409.

14. Australian Bureau of Statistics. 2016 Census QuickStats. Canberra, ACT: ABS, 2017. Available at https://quickstats.censusdata.abs.gov.au/ census_services/getproduct/census/2016/ quickstat/036?opendocument [Accessed 24 January 2020]. 